\title{
Antenatal Diagnosis of Hirschsprung Disease
}

\author{
Ashutosh Gupta $\cdot$ Anjila Aneja $\cdot$ Sanjay Mehta $\cdot$ \\ Tauqeer Syed Fazal
}

Received: 19 April 2014/ Accepted: 23 September 2014/Published online: 9 October 2014

(C) Society of Fetal Medicine 2014

\begin{abstract}
Isolated prenatally diagnosed large colonic dilatation in late third trimester raised suspicion of colonic obstruction. On further investigating, after the baby failed to pass meconium after $24 \mathrm{~h}$, with radiograph and barium enema confirmed the diagnosis of Hirschsprung disease. Even though the sensitivity and predictability of antenatal ultrasound is limited, prenatally diagnosed colonic dilatation should be investigated further to confirmation of any underlying colonic obstruction and for timely intervention to decompress the dilated loops and to avoid any life threatening complications.
\end{abstract}

Keywords Prenatal $\cdot$ Colonic dilatation $\cdot$ Hirschsprung disease

\section{Introduction}

Hirschsprung disease (HD) is one of the commonest reasons for colonic obstruction in the neonate, with an incidence of 1:5,000 live births [1]. There is an absence of intramural ganglion cells of both the myenteric and submucosal plexuses (Auerbach and Meissner plexuses,

A. Gupta ( $\square)$

Department of Fetal Medicine and Clinical Genetics, Max Super Speciality Hospital, West Block 1 Press Enclave Road, Saket, New Delhi 110017, India

e-mail: dr_ashutosh75@rediffmail.com

\section{A. Aneja}

Department of Minimal Access \& OBGYN, Fortis Memorial

Research Institute, Gurgaon, Haryana, India

S. Mehta · T. S. Fazal

Department of Radiology, Artemis Health Institute, Gurgaon, Haryana, India respectively) downstream of the dilated part of the colon, which has been recognized as the cause of the disease as early as 1940 [2].

It is characterized by large bowel distension which may begin insidiously in the late second or third trimester [3]. In the setting of antenatal colonic dilatation, sensitivity and positive predictability of ultrasound in predicting imperforate anus and Hirschsprung disease is low-in range of 8 and $15 \%$ respectively [4]. Antenatal identification of distension of the gut suggests investigation for underlying colonic obstruction, as it helps enormously by timely surgical intervention and decompression of dilated gut loops after birth [3]. Hirschsprung disease results from the failure of the parasympathetic nerve cells either to develop or to migrate to a segment of large bowel, proximally from the anus further upwards, which results in functional obstruction.

Possible association between a history of maternal hyperthermia in the first trimester and the embryonic development and migration of ganglion cells along the intestine has been reported [5]. The prenatal diagnosis of aganglionosis of the colon is extremely rare and has been reported in literature only twice and both of them presented with dilated loops of bowel and polyhydramnios [2,6]. Eight genes have been implicated in the causation of HD, out of which, RET protooncogene at 10q11.2 accounts for approximately $50 \%$ of the familial and $20 \%$ of the sporadic cases [7]. Diagnosis of HD can be confirmed after the birth of the baby by barium enema and rectal biopsy. The treatment is staged surgery (colostomy and pull-through) to remove the nonfunctioning bowel and restore bowel function. There may be other reasons for abdominal distension and failure to pass meconium in a newborn: (1) meconium ileus, resulting from cystic fibrosis; (2) anatomical intestinal malformations, ileal and colonic atresia, 


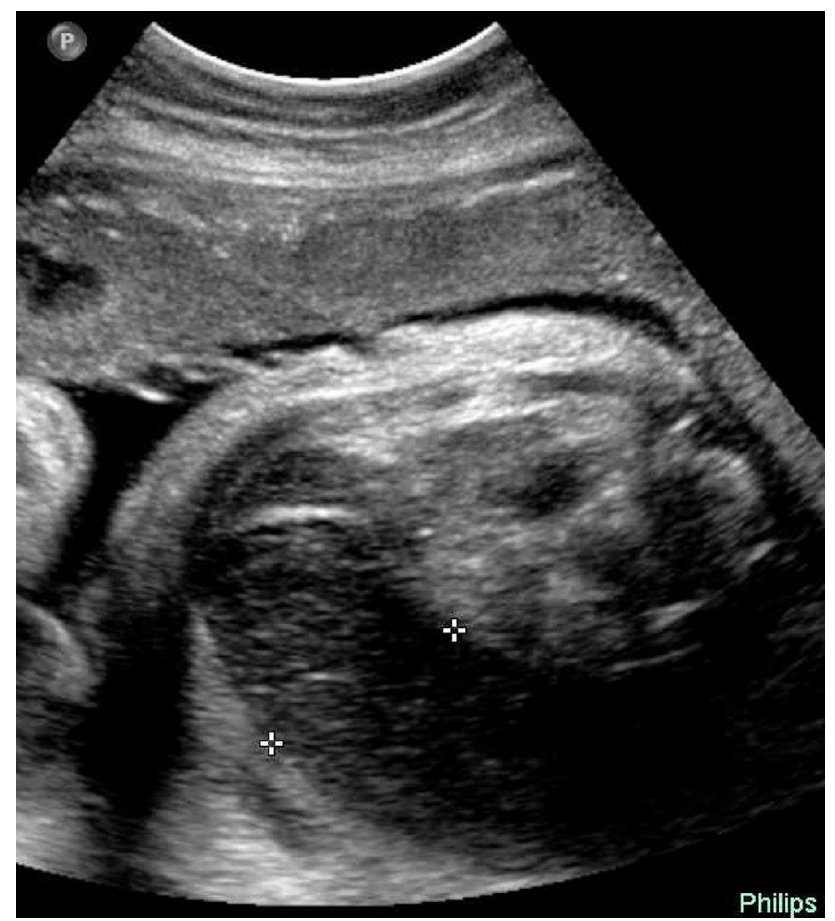

Fig. 1 Antenatally dilated? transverse colon

intestinal malrotation or duplication; (3) functional intestinal obstruction due to maternal infections or hypothyroidism.

\section{Report of Case}

A 29-year-old primigravida at 36 weeks of pregnancy was identified to have dilated large bowel loops suspected to be the transverse and ascending colon (Fig. 1). There were no other associated findings; the amount of liquor was also within the normal range. Her previous antenatal ultrasounds were unremarkable; there was no growth restriction or amount of amniotic fluid. The referring gynecologist and the attending pediatrician were sensitized regarding the finding and suspicion of colonic obstruction. The attendants were also counseled regarding watchful expectancy for passage of meconium after birth. After the baby did not pass meconium for $24 \mathrm{~h}$ of birth, a gentle probation of the rectum was undertaken for meconium staining. After inserting the nasogastric tube up to $6 \mathrm{~cm}$, no meconium staining was observed (Fig. 2). The baby was taken up for further investigations. Plain X-ray film of the abdomen of the baby revealed multiple dilated gut loops (Fig. 3), which on follow-up barium enema study, showed constricted sigmoid and descending colon suggestive of HD (Fig. 4). The baby was operated upon, which confirmed the

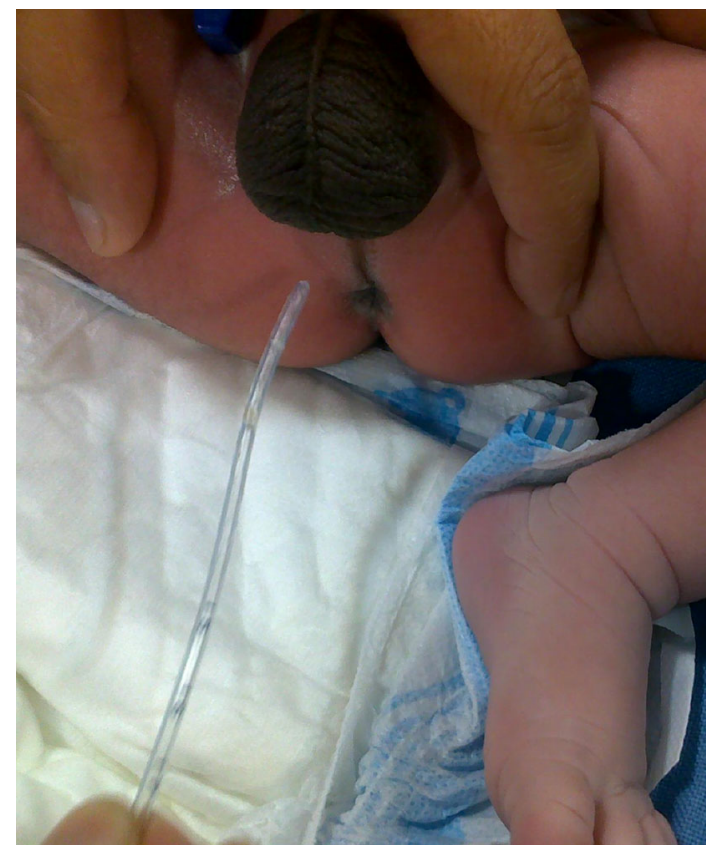

Fig. 2 Absence of meconium staining on nasogastric tube

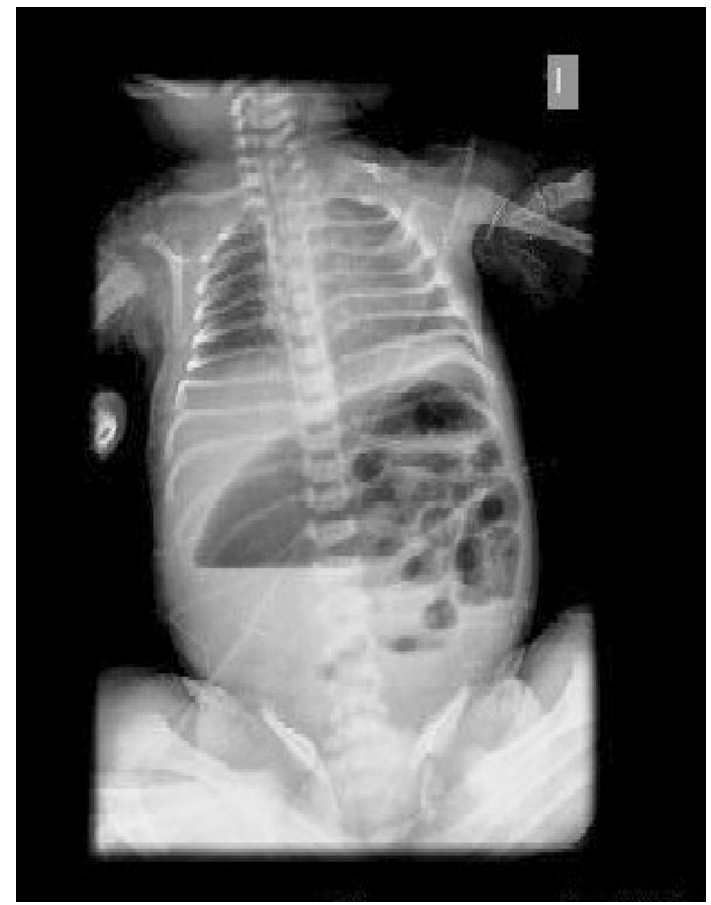

Fig. 3 Plain X-ray picture of abdomen showing massive dilatation of colonic segments

diagnosis. Initial colostomy was done to decompress the dilated loops and to prepare the baby for second, definitive (pull-through) surgery. 


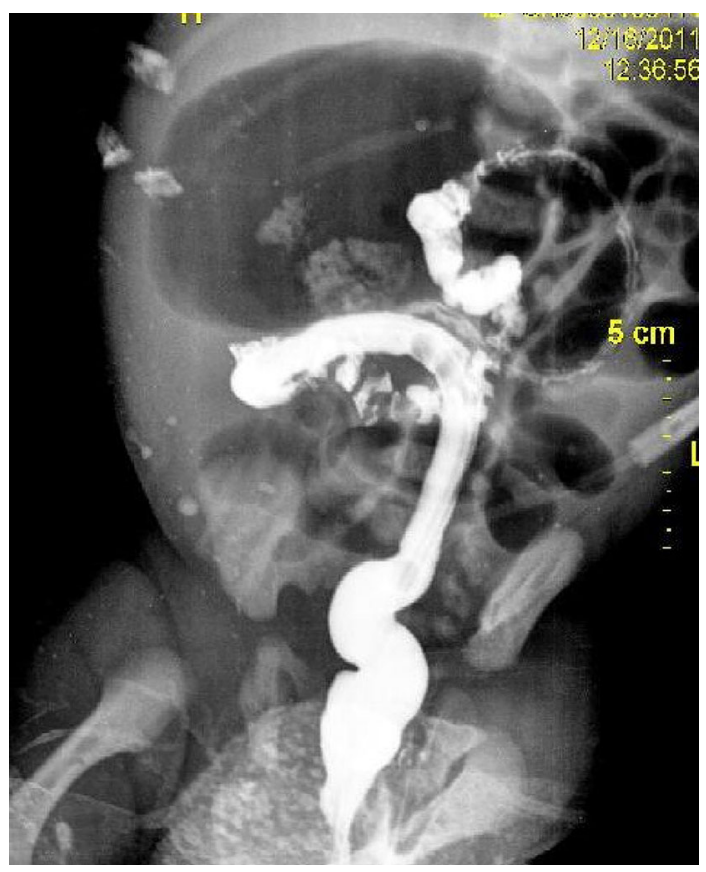

Fig. 4 Barium enema showing contracted sigmoid colon

\section{Discussion}

Two-third of the cases of HD may be diagnosed early in the neonatal period with varied symptoms like severe, continuous constipation with intermittent diarrhea and rarely with life-threatening complication such as enterocolitis [8]. Antenatal diagnosis of HD has been attempted unsuccessfully with positive predictability of around $15 \%$ [4]. Belin et al. did a prospective study of approximately 15,000 pregnancies for prenatal ultrasound identification of colonic dilatation; out of 13 suspected cases of distension, 7 were found to be normal, 2 had imperforate anus, 3 had meconium ileus and 1 had malrotation [4]. Even though nomograms for the gestation specific diameter for descending colon and rectal are available, still ultrasound modality is not able to predict accurately, HD and imperforate anus [9]. Apart from noninvasive ultrasound, prenatal diagnosis of HD has been attempted by measuring amniotic fluid disaccharidase activity in the amniotic fluid, but without much success [10].

\section{Inheritance}

Hirschsprung disease is a multifactorial disease; it may be familial; autosomal dominant with reduced penetrance has been described. It has been reported to have sex modified mutifactorial in inheritance [11]. Risk of recurrence depends upon the ganglionic growth in the colon and the sex of the first affected baby. If ganglionic growth is beyond the sigmoid colon; the inheritance may be autosomal dominant with reduced penetrance and risk of recurrence is close to $50 \%$; if smaller segment is involved then the inheritance may be multifactorial with risk of recurrence of less than $50 \%$ or autosomal recessive with recurrence risk of $25 \%$ or a sex modified multifactorial disorder (if a female with long colonic segment is affected then the risk of recurrence is higher). Overall prognosis for $\mathrm{HD}$ is very good, but a great emphasis should be placed on identifying the subtle gut dilatation in late second or even in late third trimester in the absence of associated polyhydramnios and investigating it further for confirmation.

\section{Conclusion}

In a case of dilatation of gut, small or large is detected prenatally; it should be followed-up with further investigations for the confirmation of any underlying colonic obstruction. This is necessary as the timely diagnosis and surgical intervention help in decompressing the dilated gut loops and prevent life-threatening complications like perforation and enetrocolitis. It is also helpful in risk assessment for future pregnancies and close surveillance antenatally.

\section{Conflict of interest None.}

\section{References}

1. Amiel J, Lyonnet S. Hirschsprung disease, associated syndromes, and genetics: a review. J Med Genet. 2001;38:729-39.

2. Vermesh M, Mayden KL, Confino E, et al. Prenatal sonographic diagnosis of Hirschsprung's disease. J Ultrasound Med. 1986;5(1):37-9.

3. Whitehouse F, Kernohan J. Myenteric plexus in congenital megacolon; study of eleven cases. Arch Int Med. 1948;82:75-111.

4. Belin B, Corteville JE, Langer JC. How accurate is prenatal sonography for the diagnosis of imperforate anus and Hirschsprung's disease? Pediatr Surg Int. 1995;10(1):30-2.

5. Lipson A. Hirschsprung disease in the offspring of mothers exposed to hyperthermia during pregnancy. Am J Med Genet. 1988;29(1):117-24.

6. Wrobleski D, Wesselhoeft C. Ultrasonic diagnosis of prenatal intestinal obstruction. J Pediatr Surg. 1979;14(5):598-600.

7. Parisi MA, Kapur RP. Genetics of Hirschsprung disease. Curr Opin Pediatr. 2000;12:610-7.

8. Torfs CP. An epidemiological study of Hirschsprung disease in a multiracial California population. The Third International Meetings: Hirschsprung disease and related neurocristopathies. Evian, France.

9. Zalel Y, Perlitz Y, Gamzu R, et al. In-utero development of the fetal colon and rectum: sonographic evaluation. Ultrasound Obstet Gynecol. 2003;21(2):161-4.

10. Jarmas AL, Weaver DD, Padilla LM, et al. Hirschsprung disease: etiologic implications of unsuccessful prenatal diagnosis. Am J Med Genet. 1983;16(2):163-7.

11. Amiel J, Sproat-Emison E, Garcia-Barcelo M, et al. Hirschsprung disease, associated syndromes and genetics: a review. J Med Genet. 2008;45:1-14. 